\title{
Carbon Nanotube-Based Photonic Devices: Applications in Nonlinear Optics
}

\author{
Amos Martinez and Shinji Yamashita \\ The University of Tokyo \\ Japan
}

\section{Introduction}

Carbon Nanotubes (CNTs) were first discovered by Sumio Ijima in the early 90s (Ijima et al., 1991). CNTs have since become a prominent material for an amazing breath of scientific and technological disciplines ranging from structural and material science to chemistry, biology and electronics (Dresselhaus et al., 2001). Compared to other areas of science, the study and exploitation of the optical properties of CNTs is still in its early years. In the late 1990s, various theoretical and experimental studies reported the remarkable optical properties of Carbon nanotubes (CNTs) (Kataura et al., 1999; Margulis \& Sizikova, 1998). Since then, the potential applications of CNTs have been attracting increasing attention from the photonics research community. CNTs exhibit an exceptionally high third-order optical nonlinearity and nonlinear saturable absorption with ultrafast recovery time and broad bandwidth operation. Thus, CNTs are becoming a key component towards the development of fibre lasers and nonlinear photonic devices. Despite the relative late start, photonics is now one of the research fields where CNTs are making a more significant contribution towards the development of next generation devices both from an academic and an a commercial point of view.

CNTs are structures from the fullerene family consisting of a honeycomb sheet of $\mathrm{sp}^{2}$ bonded carbon atoms rolled seamlessly into itself to form a cylinder. Single-walled CNT are nearly one-dimensional (1D) materials with a diameter ranging from $1 \mathrm{~nm}$ to $3 \mathrm{~nm}$, and a length that can go from 100s of nanometers to centimetres. The electronic properties of single-wall CNTs are governed by a single parameter named the chiral vector, which indicates the orientation of the tube axes with respect to the orientation of the honeycomb. Depending on this parameter, single-wall CNTs may behave as semiconductors or as metals. Those CNTs that behave as semiconductors exhibit a direct electronic bandgap which is directly proportional to the diameter of the nanotube. The optical absorption of CNT is determined by their electronic bandgap and broadband operation is a result of a large distribution of diameters formed during the CNT fabrication (Kataura et al. 1999). This discovery led to the now widespread use of CNTs in saturable absorption applications. Previous to that work, Margulis and Sizikoba had estimated theoretically that CNT presents a very high third order susceptibility $\chi^{(3)}$ in the order of $10^{-8} \mathrm{~m}^{2} / \mathrm{W}$. Third order susceptibility is responsible for processes such as third harmonic generation (THG), optical Kerr effect, self-focusing and phase conjugation (Margulis and Sizikoba, 1998). Materials with a high nonlinearity combined with a fast response time are desired for roles areas such as optical 
communications and information technology, where they can perform optical functions such as optical switching, routing and wavelength conversion.

CNTs have also found several other applications in photonics including nanometre-scale light sources, photodetectors and photovoltaic devices (Avouris et al., 2008). These applications rely on the properties of a single CNT and their potential applications in a field-effect-transistor (CNT-FET) configuration. Such CNT-FETs not only have great potential towards the development of future optoelectronic applications, but also offer an excellent platform to study optical phenomena in one-dimension. The focus of this chapter however, lays in the CNT applications in the areas of fibre lasers and optical communications. Those applications are based on the CNTs nonlinear optical absorption which is useful for noise suppression and passive mode-locked lasing (Set et al., 2004) and their high nonlinearity which has a great potential towards the implementation of optical switches (Liu et al., 1999; Chen et al., 2002). These applications rely on using a large number of CNTs and their combined optical properties. During their bulk fabrication, a large number of single-walled CNT with different tube diameters and different electrical properties are created. For most applications, the lack of ability to fully control the diameter and chiral vector of the CNTs during fabrication is a drawback. For optical fiber devices on the contrary, it is auspicious that the current fabrication processes produces a distribution of single-wall CNTs with different diameters, some behaving as a semiconductor and some as metals. The role of the semiconducting CNT is to provide the saturable optical absorption, which is proportional to the diameter of each individual CNT. The wide distribution of CNT's diameters provides broadband operation and the metallic CNTs also play an important role towards reducing the recovery time.

This chapter is structured as follows; in section 2, the physical mechanisms that are accountable for the exceptional optical properties of carbon nanotubes (CNTs) will be described. In section 3, we will describe the various methods that have been proposed for the fabrication of CNT-based photonic devices and introduce the relevant parameters that must be taken into account while preparing a CNT-based device for different applications. In section 4 we will review the most significant advances reported to date regarding passive mode-locked lasing, which is arguably the application were CNTs have made their most significant contribution so far. In section 5, we will investigate the potential applications of CNTs in optical switching devices by four-wave mixing and polarization rotation and discuss the requirements that would need to be met before such devices can be considered for commercial applications. Finally, in section 6, we will discuss the future of CNT-based photonic devices in the context of becoming an integral part in the fast developing fiber laser technology and the challenges ahead, such as the advent of graphene as an alternative to CNTs and semiconductor saturable absorbers (SESAMs) for many applications and the needs for improved robustness and long term stability of CNT-based photonic devices.

\section{Optical properties of CNT}

The original interest to apply CNTs in photonics started in the late 1990s soon after Margulis et al. reported the first theoretical study on the very high third order nonlinearity of CNT and Kataura et al. reported for the first time the nonlinear optical properties of single-wall CNT. Most applications in photonics require single-wall CNT, hence from here on we will refer to those single-wall CNTs simply as CNTs. The optical properties of CNTs are linked closely to their structural and electronic properties and are fully governed by one parameter, its chiral vector, which describes how the carbon-atom honeycomb is organized with respect 
to the CNT axis. Depending solely on this parameter, CNTs may behave as a direct bandgap semiconductor or as a metal.

\subsection{Electronic structure}

The electronic properties of a CNT are determined by its chiral vector. This vector describes how the graphene sheet is rolled when forming the nanotube. The chiral vector, $C_{h}$ is expressed by two real space unit vectors $a_{1}$ and $a_{2}$ and two integers $\mathrm{n}$ and $\mathrm{m}$ as shown in equation 1 :

$$
C_{h}=n \bar{a}_{1}+m \bar{a}_{2}
$$

Graphene is a semimetal, when the single-layer graphene is rolled to form a tube an extra level of confinement into a quasi-1D structure is added. CNTs behave as metals if the subtraction of the integer values $n$ and $m$ is an integer multiple of $3(n-m=3 k)$ otherwise it is a semiconductor $(n-m \neq 3)$. Hence, statistically we can conclude that $2 / 3$ of CNTs will be semiconductors, while $1 / 3$ will be metallic CNTs. Saturable absorption is a result of the excitonic absorption in semiconductor nanotubes (Saito et al., 1992).

The density of states of a semiconducting CNT can be numerically calculated. In figure 1, the density of state of two semiconducting CNTs with different diameters is shown. In fact, the electronic density of states of CNT, originates from the "slicing of the graphene density of states due to the quasi-1D confinement of the electrons, which leads to the presence of the characteristic Van Hove singularities observed in their electronic densities of state (DOS) of the two CNTs shown in figure 1 (from Yamashita et al., 2007). Semiconducting CNTs absorb photons in the same way an ordinary direct bandgap semiconductor would do when the energy of the photon is equal to the bandgap of the CNT. The CNT's bandgap is inversely proportional to the diameter of the tube. It is particularly fortunate that the diameter of a CNT typically ranges from $1 \mathrm{~nm}$ to $2 \mathrm{~nm}$, which leads to an optical absorption that overlaps the $1550 \mathrm{~nm}$ window commonly used for fiber optic technologies.

Figure 1(c) shows the characteristic relation of the energy bandgap as a function of the diameter of the semiconductor CNT. This figure is known as the Kataura plots and indicates the allowed band transmissions based on the diameter of the tube.
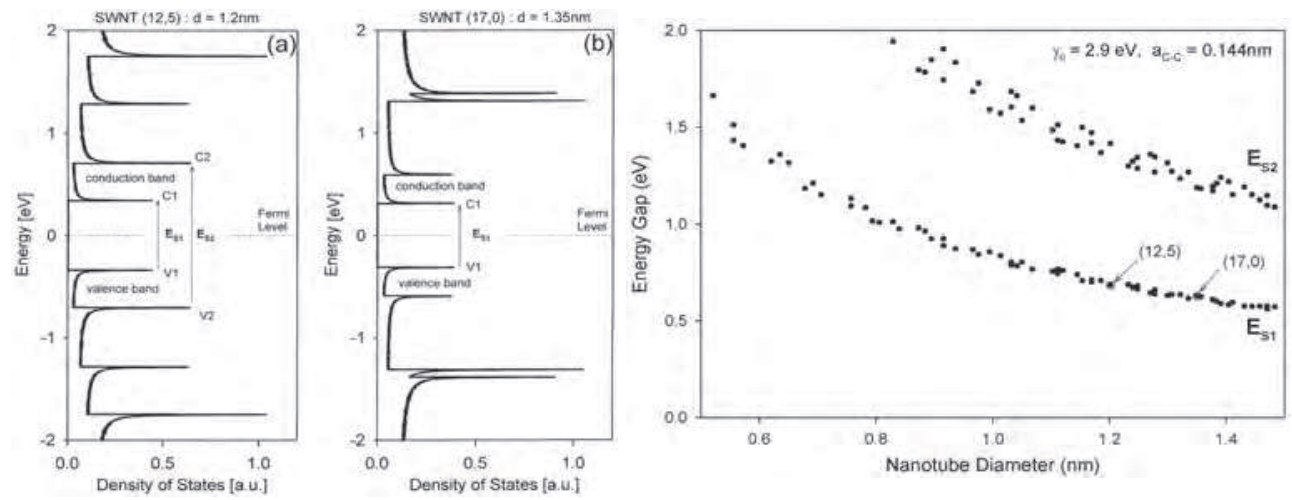

Fig. 1. (a) and (b) show the calculated electronic density of states (DOS) of two CNTs with tube diameters $1.2 \mathrm{~nm}$ and 1.35, respectively. Both DOS exhibit sharp singularities caused by the characteristic 1D structure of CNT (c) shows the relation between the nanotube's diameter and the allowed electron transitions. Figure taken from (Yamashita et al., 2007) 


\subsection{Saturable absorption}

The optical absorption of CNTs is of saturable, intensity-dependent nature, it is, therefore, a suitable material to employ for passively mode-locked laser operation. Passive modelocking is achieved by incorporating an intensity-dependent component into the optical system, this is generally a saturable absorber which absorbs the incoming light linear up to a given threshold intensity, after which it saturates and becomes transparent. Such saturable absorbers discriminate in favor of pulse formation over continuous wave lasing.

Current processes for the bulk fabrication of CNTs do not allow complete control over the chirality and diameter of the fabricated CNTs. Instead, a combination of metal and semiconductor CNTs and multi-wall CNTs are produced during fabrication. Nevertheless, researchers are now able to separate CNTs according to their chiral vector through post-fabrication processes. In actual fact, depending on the application, a wide distribution of CNT diameters or a very narrow distribution may be beneficial. Hence, the ability to control the mean diameter and diameter distribution through the fabrication, purification and separation processes is likely to further extend the applications of CNTs in photonics. Figure 2(a) shows the typical absorption of a suspension of CNT fabricated by the high pressure carbon monoxide method (HiPCO) and measured by a spectrometer, Absorption peaks rise from the inter Van Hove transitions between the different semiconductor and metal transitions and can be clearly identified in the spectrometer measurements.

For ultrashort pulse generation, a saturable absorber with a fast recovery time is required for stabilizing laser mode locking, while a slower recovery time could facilitate laser selfstarting. Ultrafast recovery times of few 100s of femtoseconds have been reported making CNTs a suitable material for ultrashort pulse generation. But it is worth noting that, the recovery time is not so fast if we consider only isolated semiconducting CNTs, in this case, the recovery time is in the order of 30ps (Reich et al., 2004). The ultrafast response time of CNTs is based on bundle and entanglement of semiconducting and metallic CNTs because electrons which are excited by photons in semiconducting CNTs tunnel and couple to metallic CNTs, resulting in ultrafast recovery time of semiconducting CNTs. The combination of fast and slow processes within the CNT distribution, allows CNT to be a very efficient saturable absorber both to self-start the mode-locking operation and to produce ultrashort pulses. In addition, the inability to fully control the diameter of the CNTs during their growth is advantageous for the implementation of fiber lasers. In fact, the wide distribution of CNT diameters is responsible for the very broadband operation of CNTbased saturable absorbers. This is one of the key advantages of CNT-based devices, as has been demonstrated by Kivistö et al. achieving passive mode-locked operation not only in the $\mathrm{C}$ and $\mathrm{L}$ band but covering also the wavelength range from $1 \mu \mathrm{m}$ to $2 \mu \mathrm{m}$ using a single CNT saturable absorber (Kivistö et al., 2009).

In figure 2, the absorption characteristics of CNT-devices are summarized. Figure 2(a) shows the characteristic optical absorption properties of a CNT dispersion, the characteristic absorption peaks generated by the Van Hove transitions can be clearly observed. Figure 2(b), depicts the normalized saturable absorption, from the data in Figure 2(b) we can determine three parameters that together with the response time give us an indication of the applicability of CNTs for passive mode-locking applications. Those parameters are the nonsaturable losses $\left(\mathrm{a}_{\mathrm{ns}}\right)$, the modulation depth $\left(\mathrm{a}_{0}\right)$ and the saturation intensity $\left(\mathrm{I}_{\text {sat }}\right)$ fitted using a saturable absorber model, based on equation 2 (Wang et al., 2008). 


$$
\alpha(I)=\frac{\alpha_{0}}{1+I / I_{\text {Sat }}}+\alpha_{n s}
$$
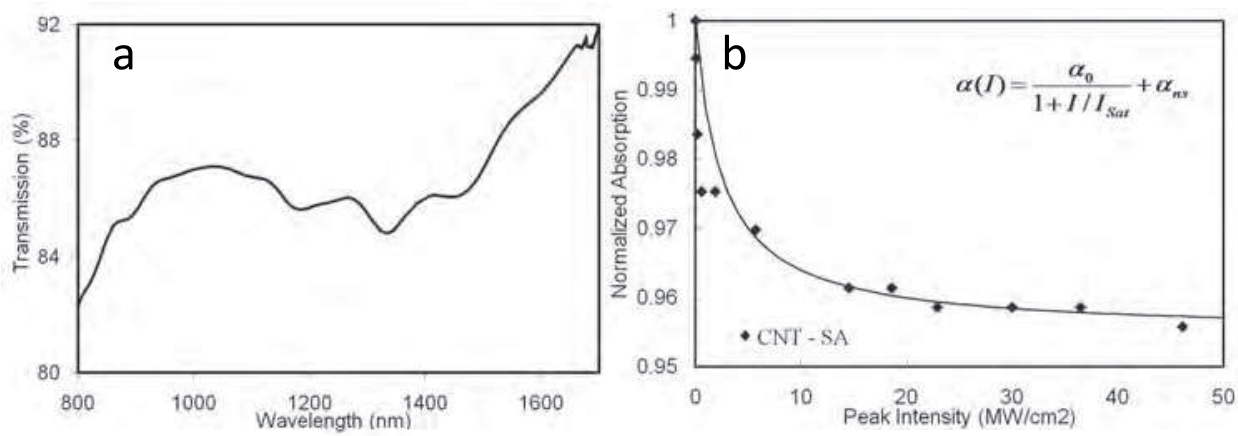

Fig. 2. Characterization of the optical absorption of CNT. (a) Characteristic broad optical absorption of a CNT solution measuredby an spectrometer. (b) Normalized absorption, the modulation depth and saturation fluence are in this case, $5 \%$ and $3 \mathrm{MW} / \mathrm{cm}^{2}$.

\subsection{Third order nonlinearity}

The first theoretical studies on the third order nonlinearity of CNTs appeared in 1998 when Margulis and Sizikova reported an estimated nonlinear refractive index coefficient, $\mathrm{n}_{2}$ as high as $2 \times 10^{-8}\left(\mathrm{~m}^{2} / \mathrm{W}\right)$. To put this theoretical value into context, $\mathrm{n}_{2}$ for the silica glass is $3 \times 10^{-20}\left(\mathrm{~m}^{2} / \mathrm{W}\right)$ and chalcogenide glass, which is recently receiving much attention as a suitable material for the fabrication of highly nonlinear fibers has a $\mathrm{n}_{2}$ value of $2 \times 10^{-18} \mathrm{~m}^{2} / \mathrm{W}$. Table 1 shows typical third order susceptibility values for various materials used in nonlinear optics.

\begin{tabular}{|c|c|c|c|c|c|c|}
\hline & $\begin{array}{c}\text { Silica } \\
\text { glass }\end{array}$ & $\begin{array}{c}\text { Chalcogenide } \\
\text { glass }\end{array}$ & $\begin{array}{c}\text { Poly- } \\
\text { diacetylene } \\
\text { (PDA) }\end{array}$ & GaAs & Si & CNT \\
\hline $\mathrm{n}_{2}\left(\mathrm{~m}^{2} / \mathrm{W}\right)$ & $3 \times 10^{-20}$ & $2 \times 10^{-18}$ & $1 \times 10-15$ & $1 \times 10-10$ & $4 \times 10-18$ & $2 \times 10-12$ \\
\hline $\begin{array}{c}\text { Response } \\
\text { time }(\mathrm{ps})\end{array}$ & $\sim 0.01$ & $\sim 0.01$ & $\sim 0.01$ & $\sim 100$ & 0.01 & $<1$ \\
\hline Absorption & small & small & small & large & small & large \\
\hline
\end{tabular}

Table 1. Comparison for the nonlinear refractive coefficient $\left(\mathrm{n}_{2}\right)$, the response time and the optical absorption for various materials commonly used for nonlinear optics.

The optical nonlinearity of CNT comes from the 1D motion of the delocalized $\Pi$-band electrons at a fixed lattice ion configuration. These estimations attracted a great deal of attention since third order nonlinearity is responsible for phenomena such as third harmonic generation (THG), optical Kerr effect, self-focusing and phase conjugation. As a result, third 
order nonlinear materials can be considered for optical functions such as optical switching, routing and wavelength conversion. The difficulty is that for most third order nonlinear optical materials, the third order susceptibility was too low for practical applications; the extremely high $\mathrm{n}_{2}$ of CNT opened a new potential application for CNT.

Optical nonlinearity is due to optical intensity dependent nonlinear response of dielectric materials. When the optical intensity of propagating light in a dielectric material is low, stimulated polarization is linearly proportional to the optical intensity. However, at high optical intensities, the stimulated polarization, $\mathrm{P}$, exhibits a nonlinear response to the optical electric field, E, as described by equation 3 (Agrawal, 1995);

$$
\mathrm{P}=\varepsilon_{0}\left(\chi^{(1)} \cdot \mathrm{E}+\chi^{(2)} \cdot \mathrm{EE}+\chi^{(3)}: \mathrm{EEE}+\ldots\right)
$$

Where $\chi^{(1)}, \chi^{(2)}$ and $\chi^{(3)}$ correspond to the linear, second and third order susceptibility. It has been reported that some types of CNTs present a high $\chi^{(2)}$, however there is not much active research on exploiting this property. On the other hand, the high values of $\chi^{(3)}$ calculated theoretically and experimentally are of great interest. In most cases there is a trade-off between high optical nonlinearity and recovery. For instance, optical fibers generally exhibit low nonlinearity and a fast response time while semiconductor based devices generally operate with a high nonlinear coefficient and low response time. Remarkably, CNT devices offer a very high nonlinear coefficient combined with a fast response time due to their nanostructures and the Van der Waals induced bundling. In CNTs, there are two main contributions to their relaxation processes; a non-radiative relaxation such as multi-phonon emission through electron-phonon coupling and an excited electron tunneling to neighbouring CNTs. Since the CNTs whose electrons are excited and those whose electrons are relaxed are different, fast relaxation process in the order of 100 s of femtoseconds combined with an extremely high nonlinear coefficient can be obserbed in real CNT devices. Of course, the technological implications of using such material would be enormous since it opens the door for the fabrication of nonlinear devices with miniature size and lower power consumption leading to further integration and compactness of CNT-based nonlinear devices. However, their implementation presents numerous challenges; in particular in order to exploit the third order nonlinearity of such devices CNT, the interaction length between the $\mathrm{CNT}$ and the optical field must be increased. Unlike silica and chalcogenide glasses, CNTs absorption of the incident light is very high, this combined with the high scattering of CNT bundles complicates the fabrication of nonlinear devices with longer interaction lengths required to increase the nonlinearity of the device. In section 3.3, the current efforts towards fabrication CNT-fiber devices with increased interaction length suitable for the exploitation of the nonlinear susceptibility of CNT for optical switching applications is described. Section 5, describe the few experimental demonstrations of optical switching by polarization rotation and four wave mixing (FWM) using CNT-based devices with long interaction lengths.

\section{Fabrication of CNT-based photonic devices}

Since 2003, several laser configurations and methods in which to integrate the CNTs into the fiber have been considered, each to suit different requirements. Initial devices were fabricated by spraying a solution of CNT in a substrate, mirror or fiber ferrule (Set et al., 2004; Yamashita et al., 2005) or by direct growth of the CNT in a substrate using chemical 
vapor deposition (CVD) (Song et al., 2007). In order to spray the CNTs, they are first independently dispersed into a solvent, such as Dimethylformamide (DMF) solvent by ultrasonification. This is necessary in order to separate individual CNTs and breaking of bundles of CNT that are formed due to van der Waal forces. Once efficient dispersion is achieved the solution is subjected to centrifugation so the remaining agglomerated CNTs can be separated and discarded. The CNTs can then be sprayed into the substrate surface, using a set-up such as shown in figure $3(\mathrm{a})$.

While the spray method is suitable to fabricate CNT-Saturable absorbers, the usage of CNT in order to coat the fiber is very high. Kashiwagi et al. and Nicholson et al. developed a method where by using an optical deposition technique CNTs could be deposited efficiently only in the core area of the fiber, reducing the CNT solution usage dramatically (Kashiwagi et al., 2007; Nicholson et al., 2007). The experimental set-up used for the optical deposition of the samples is shown in Fig.3 (b). Deposition is achieved by immersing a flat fiber connector into the solution of CNT on DMF and launching a high-power light into the fiber. The optical power required to achieve optical deposition is dependent on a number of parameters, including the concentration levels of the solution, and how well the CNTs have been dispersed on the solvent.
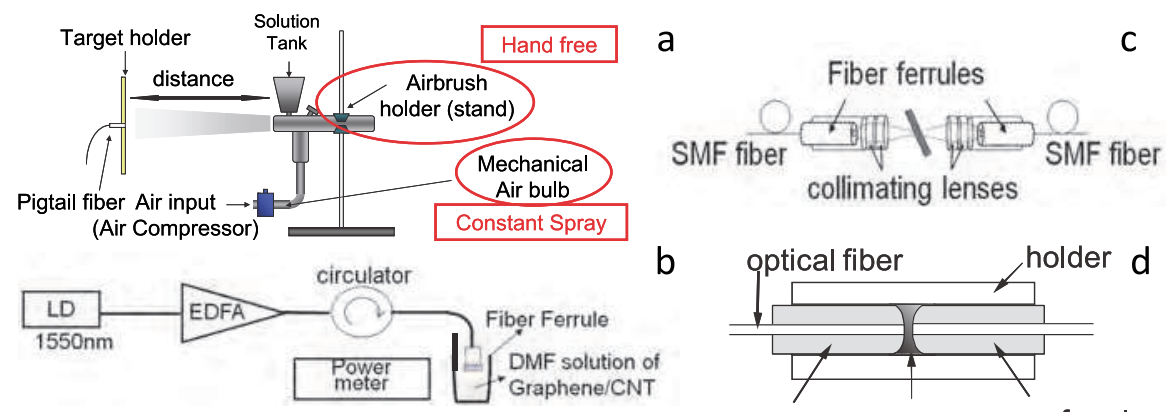

b

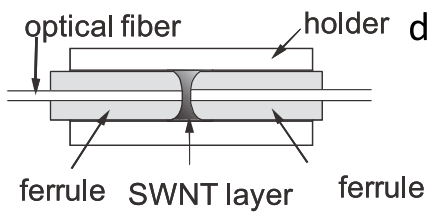

Fig. 3. Experimental set-up for the fabrication of CNT saturable absorbers (a) using the spray method (b) using the optical deposition method. (c) and (d) show original configurations used to integrate the CNT into the optical system.

The physical mechanisms responsible for the optical deposition consist of a combination of optical trapping and heat convention effects. The first demonstrated saturable absorber incorporating CNT consisted of a glass plate with sprayed CNT on its surface such as the one shown if figure 3(c) however this approach required free space alignment and this led to increased insertion losses and lower stability. It was soon recognized that saturable absorbers fabricated directly into the fiber-end by either the spray method or the optical deposition method would benefit from more stable operation in a simpler and highly integrated configuration (Fig.3.(d)). Using these two simple methods a great diversity of devices have been fabricated. The saturable absorber consists on the CNT-coated fiber ferrule butt-coupled between two fiber ferrules and inserted into an all fiber ring cavity laser. Using this configuration, short pulse operation can be achieved, with pulse durations in the order of 100 s of femtoseconds. Figure. 4 shows the microscope picture and scanning electron microscope picture of a CNT-coated fiber-end by optical deposition. 

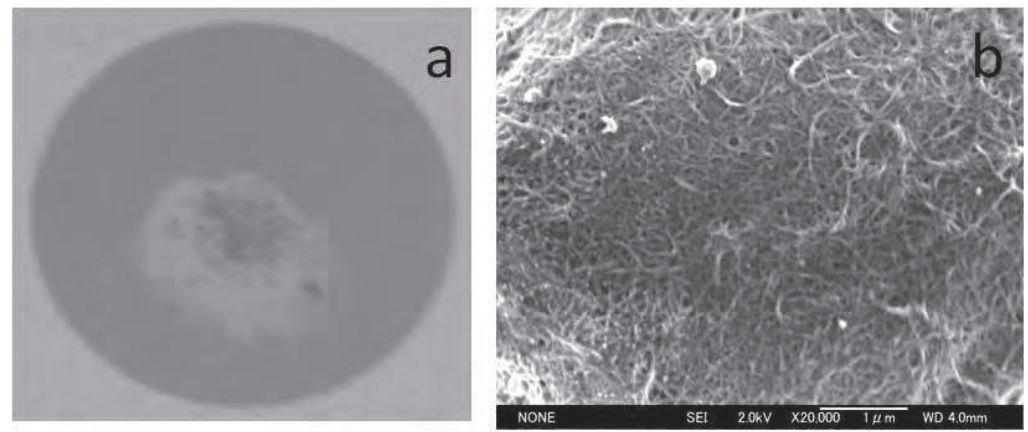

Fig. 4. (a) Microscopic picture of a fiber with optically deposited CNT, (b) Scanning electron microscope image of the same sample.

\subsection{Fabrication of CNT-polymer composites}

The first demonstrations of CNT-based photonic devices were fabricated by spray-coating a substrate with CNTs suspended in a solvent or by directly synthesizing a CNT film on a substrate. While those reports confirm the feasibility and great potential of this novel technology, they also showed the stringent limitations of those fabrication techniques. CNTbased devices could only be formed as thin films, and that limited the interaction length between the propagating light and the CNTs therefore, hindering the efficient exploitation of their nonlinear properties. In addition, those devices suffer degradation owing to the permanent contact with the external environment. In order to fully utilize the nonlinear optical properties of CNT, these shortcomings needed to be addressed. Researchers concentrated their efforts on finding an optimum host material in which to disperse and embed the individual CNTs. Polymers were identified as such suitable host material since they are transparent at optical communications wavelengths and have a structural composition that allows uniform dispersion of the CNTs. There have been numerous reports of composites consisting of CNT embedded in polymers such as polyvinylalcohol (Rozhin et al., 2006), polyamide (Schibli et al., 2005), carboxymethyl cellulose (CMC) (Tausenev et al., 2007) or polymethyl-methacrylate and polysterene (Nakazawa et al., 2006). Those devices were used as saturable absorbers in various passively mode-locked laser configurations. The main challenge initially was to disperse efficiently the CNT in the polymer. CNTs tend to agglomerate together forming bundles of tens to hundreds of individual carbon nanotubes, the dispersion of such bundles is a key factor to fabricate efficient devices. The bundle breaking process is generally carried out by subjecting the CNTs to an ultrasonic bath in the presence of a solvent. Most approaches to fabricate CNT-doped polymers consist on dispersing the CNTs in a solution of polymer. As a result, the process of solvent evaporation is inevitable which limits the composite dimensions. Furthermore, the dispersion takes place in a rather viscous solution complicating the breaking up of bundles and efficient dispersion of the CNT. Creating bulk CNT-polymer compounds is important since it provides a platform to fabricate complex photonic structures such as CNT-doped fibers, and such structures would allow the efficient exploitation of the nonlinear saturable absorption and third order nonlinearity of CNTs. An alternative method developed by S. Uchida consists on adding the CNT to a solution of methyl-methacrylate (MMA) monomer with a polymerization initiator. CNTs are mixed and dispersed by ultrasonication in methyl- 
methacrylate (MMA) monomer prior to and during the polymerization process, thus it does not require the use of solvents (Uchida et al., 2009). The CNTs are dispersed in a low viscosity liquid (MMA) rather than a dissolved polymer, hence CNT bundle separation is facilitated. After the thermally-aided polymerization we obtain a bulk state of evenly dispersed CNT-doped PMMA. Using this method, bulk CNT-embedded PMMA with a tangible improvement in the dispersibility of the CNTs and with added flexibility on device design can be produced. In Figure. 5, polished disks from bulk CNT-PPMA composites are shown, the effect of adding dopants such as DPS and BEN was also investigated with results indicate that these dopants play the role of CNT dispersion stabilizer and thus improving the optical properties of the CNT-PMMA composite. Nowadays, the fabrication of CNTpolymer composites has advanced greatly and the evolution an current state of CNTpolymer composites has been reviewed in detail by T. Hasam (Hasam et al. 2009).

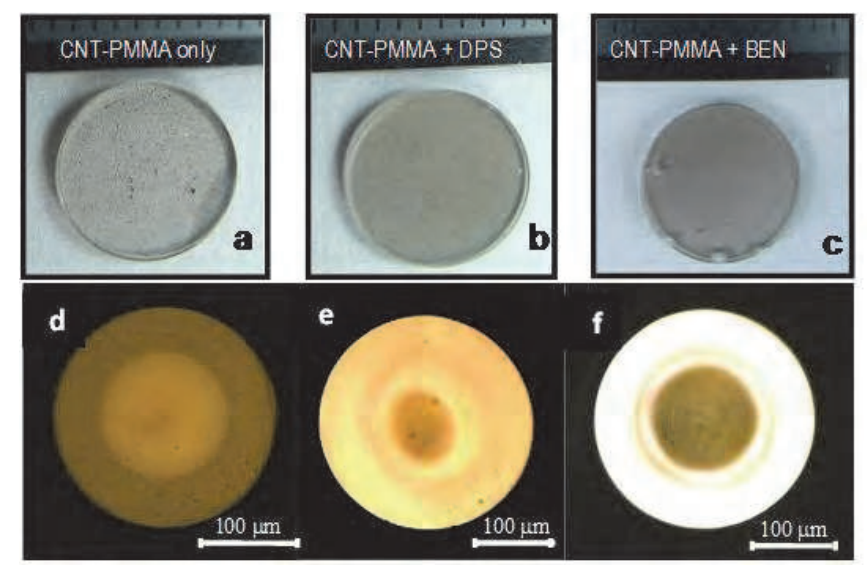

Fig. 5. Bulk fabrication of polymethyl-methacrylate (PMMA) doped with CNT under the presence of different dopants (a) no dopant, (b) diphenyl sulfide (DPS) and (c) benzyl benzoate (BEN). (d), (e) and (f) correspond to CNT-doped polymer optical fibers fabricated using PMMA (Uchida et al., 2009)

\subsection{CNT based devices for enhanced nonlinear applications}

The nonlinearity in an optical material is proportional to the interaction length between the nonlinear material (i.e. carbon nanotubes) and the propagating optical field. Hence, such nonlinearity can be enhanced by increasing the length of interaction between the CNTs and the propagating optical field. Numerous approaches have been considered for this purpose by employing devices such as D-Shape fibers (Song et al., 2007) tapered fibers (Kieu et al., 2009), hollow core fibers (Choi et al, 2009), micro-slot fiber devices (Martinez et al., 2010) and planar waveguides (Kashiwagi et al., 2007). These approaches involve evanescent coupling, where the CNTs are placed adjacent to the optical waveguide. Using these approaches the interaction length can be significantly increased and only the tail of the pulse propagating outside of the core interacts with the CNT. This means that the CNT are subjected only to a small fraction of the peak power propagating through the device. Thus, the device can operate at higher powers before damage is observed. Using such approach the nonlinear response increases due to the longer interaction, this increase in nonlinearity is somehow hampered by the lower intensity of 
the light that effectively interacts with the CNT (note that the nonlinearity is also dependent on the light intensity). Other approaches based on evanescent and direct interaction have been demonstrated that provide increased interaction length with the CNTs. Recently, the fabrication of CNT-doped polymer optical fibers (POF) has been demonstrated. By doping the POFs core with CNTs, highly integrated devices can be in principle integrated, which will allow the efficient utilization of the optical properties of the CNTs (Uchida et al., 2009). However, this CNT-doped POF suffers from high insertion and coupling losses due to been multimode, large

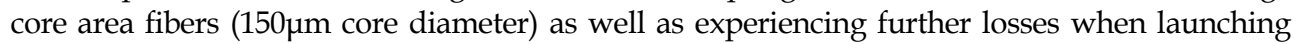
the light into the fiber. An alternative approach based on directly injecting a solution of CNT into a micro-channel fabricated into a standard optical power has been proposed (Martinez et al., 2010). This method offers the means to achieve a highly integrated and compact configuration, while ensuring the stability and low insertion losses by directly fabricating the saturable absorber within the optical fiber. In Figure 6, various device configurations designed to increase the interaction length and nonlinearity of CNT devices are depicted.

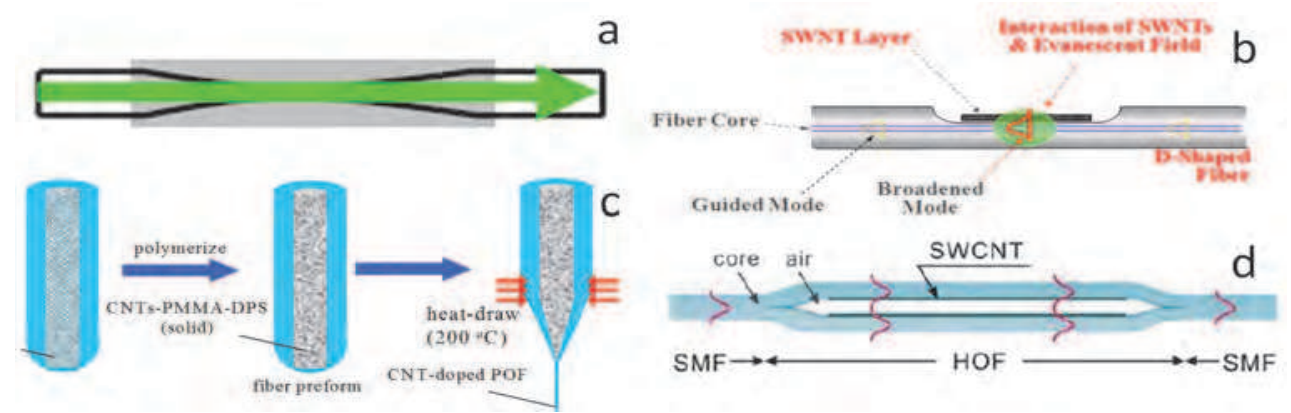

Fig. 6. (a) CNT-polymer coated taper fiber (Kieu et al., 2007), (b) D-shape coated with CNT (Song et al., 2007), (c) Polymer optical fiber doped with CNT (Uchida et al, 2009), (d) Hollow optical fiber filled with CNT (Choi et al., 2009)

In addition, to the length of interaction, the spray method and the optical deposition method present some drawbacks associated to them. In general, after spray or optical deposition, the CNTs are randomly oriented and entangled. The CNT optical absorption is highly anisotropic, and hence the direction of the propagating field with respect to the CNT axis strongly affects the nature of the interaction. Since the CNTs are randomly dispersed, only a fraction of them would contribute to the device operation. There is however a fabrication technique based on low-temperature alcohol catalytic chemical vapor deposition (CVD) method which not only can directly synthesize high-quality SWNTs onto the substrates, but also enables the fabrication of vertically aligned CNTs. This fabrication technique in conjunction with a D-shape fiber configuration has been used for the fabrication of highpulse energy, high efficiency fiber lasers (Song et al., 2007)

\section{Passive mode-locked lasers using CNT saturable absorbers}

Passive mode-locked lasers are, nowadays, present in many industrial and scientific applications that range from micromachining and microscopy to optical communications. Passive mode-locking is achieved by incorporating an intensity-dependent component, 
generally a saturable absorber, into the optical system. Broadly speaking, this component absorbs the incoming light linearly up to a certain threshold intensity. Once this intensity is reached, the saturable absorber saturates and becomes transparent. Such saturable absorbers discriminate in favor of pulse formation over continuous wave lasing. Passive mode-locking is generally preferred to active mode-locking techniques due to its simplicity and the ability to produce transform-limited pulses without the need for any external active devices such as modulators. The optical absorption of CNTs is of saturable, intensity-dependent nature and hence it is a suitable material to employ for passively mode-locked laser operation with CNT-based saturable absorbers rapidly gaining ground on more conventional approaches to achieve saturable absorption such as the semiconductor-based saturable absorbers mirrors (SESAM) and Nonlinear Optical Loop Mirrors (NOLM). At this moment, SESAMs are still the prevalent device in commercial passively mode-locked lasers, however their fabrication process is complex requiring clean room facilities and specific fabrication for each operational wavelength. The recovery time of a saturable absorber limits the laser repetition rate, SESAMs require an additional ion implantation fabrication step to reduce the recovery time from the nanosecond regime into the picosecond regime. On the other hand, CNTs exhibit a sub-picosecond recovery time, broadband operation, compatibility with fiber, a small foot-print and simple fabrication. In addition, CNT-based saturable absorbers can operate in either a transmission-mode or reflection-mode. Hence, there are several reasons why researchers are starting to favor CNT-based devices over the more established SESAMs for commercial applications.

\subsection{Passively mode-locked fiber lasers}

Passively mode-locked fiber lasers are nowadays capable of producing ultrashort, high power pulses with high beam quality, efficient heat dissipation and very robust operation in a compact configuration. Hence fiber laser devices are sought for material processing, supercontinuum generation, optical frequency metrology and biomedical applications where they are a more cost-effective and viable solution than their bulkier solid state laser counterparts (Fermann and Hartl, 2009). For fiber laser technology, however, there are still challenges to be met in order to compete in a level field with solid state lasers in particular in terms of the shortest available pulse durations and highest available pulse energies. In order to meet these demands, passively mode-locked laser technology is in the midst of a fascinating evolution striving towards higher powers, shorter pulses and improved stability. This evolution is been fueled by the development of novel fiber designs and model-locking mechanisms such as stretched-pulse mode-locking (Tamura et al., 1993), and the dissipative soliton regimes (Chong et al., 2006). CNTs are becoming an integral building block for many of those advances.

Since 2003, when the first demonstration of a fiber laser passively mode-locked by CNTs was reported, CNT-based devices have been demonstrated in multiple configurations and for all the relevant operational wavelengths ranging from $1 \mu \mathrm{m}$ to $2 \mu \mathrm{m}$ (Kivistö et al., 2009). Initially, all the fiber laser reports employing CNTs operated in the slightly anomalous dispersion regime. Thus, these lasers operated in the robust and highly stable solitonregime. Soliton-like fiber laser operation is capable of producing highly stable, close to transform limited (with a very low chirp), hyperbolic-secant pulse shapes with a subpicosecond pulse duration, by balancing out the chromatic dispersion of the cavity with the accumulated Kerr-nonlinearity in the fiber. Soliton-like pulses are very robust, but the pulse 
energy can hardly be scaled up, since increasing the intra-cavity power leads to increased nonlinearity consequently the dispersion and nonlinearity balance cannot be maintained. Furthermore, with this mode-locking regime pulse duration as short as a few hundreds of femtoseconds can be produced but it is difficult to further reduce the pulse duration. Figure 7 , shows the typical configuration of a fiber laser operating in the soliton regime, and its characterization in terms of optical spectrum, pulse duration and rf signal. The narrow peaks observed in the optical spectrum trace are known as Kelly sidebands, they are common features of fiber lasers operating in the soliton regime and originate from resonant coupling between the soliton and weak co-propagating dispersive waves.
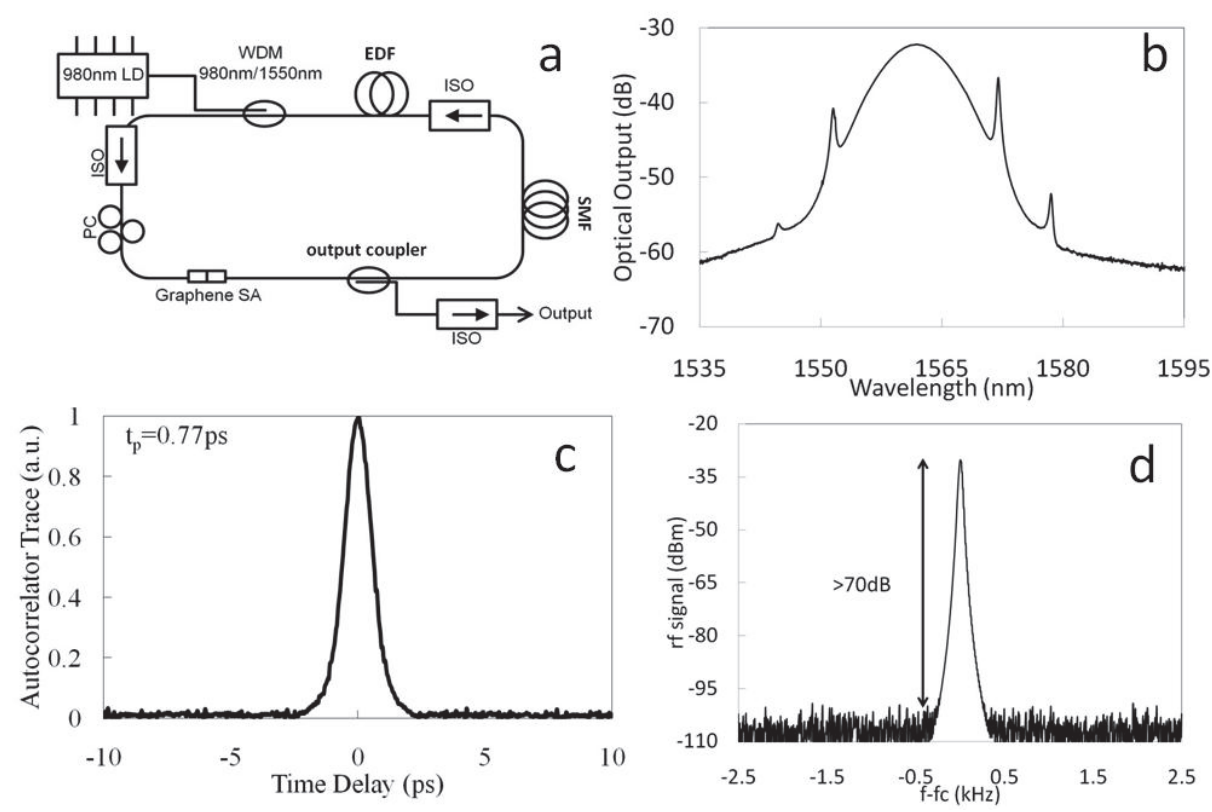

Fig. 7. Ring cavity fiber laser: (a) set-up (b) optical spectrum (c) autocorrelator trace (d) rf signal.

In recent years, researchers have also demonstrated other mode-locking regimes employing CNT saturable absorbers. For instance, stretch-pulse mode-locking, combines a section of fiber with positive dispersion and a section with negative dispersion, as a result the pulse "breaths" within the cavity, and it is chirped in certain sections of the fiber and then recompressed. The laser output in this regime is Gaussian and the operation in this regime, has several advantages with respect to soliton regime since shorter pulses and higher powers can be achieved and the Kelly sidebands can be suppressed (Kieu et al., 2009; Sun et al., 2010). Another laser regime is the dissipative soliton regime. In this case, the fiber in the cavity has positive dispersion (often used for Yb-fiber Lasers), chirp grows continuous through the fiber leaving the gain filtering mechanism and the saturable absorber to limit the spectral broadening of the pulse. Evidently, the output is strongly chirp, but this chirp is linear so it can be easily compensated outside the cavity (Kelleher et al., 2009; Sun et al., 2009). 


\subsection{Other laser configurations}

CNTs can be used to coat a mirror. Such mirror can then be used to operate in a laser in a reflection-mode. This can be applied to the fabrication of solid state lasers, where by using spin coating a CNT-polymer can be deposited over the solid state laser mirror (Schmidt et al., 2008). It is also useful to achieve short cavity lasers with multi-gigahertz repetition rates. In fact, the highest ever reported fundamental repetition rates for a fiber laser employed a phosphosilicate fiber codoped with erbium and ytterbium with a CNT-coated fiber ferrule mirror and reached a repetition rate as high as $20 \mathrm{GHz}$ (Martinez et al., 2011). The laser setup and output characteristics are summarized in Figure 8. Similarly, High repetition rate, passively mode-locked lasing by using an ultrashort cavity with a gain media consisting of a semiconductor optical amplifier (SOA) has been demonstrated, the repetition rate in this case was 17.2GHz (Song et al., 2007). In ring cavity fiber lasers, the long gain fibers can compensate for the insertion losses of the saturable absorber. However, for this type of applications, the requirements for the saturable absorber are much more stringent, with excessive non-saturable losses truncating the modelocked operation and were optical power and heat damage must be carefully taken into account in the design of the saturable absorber.
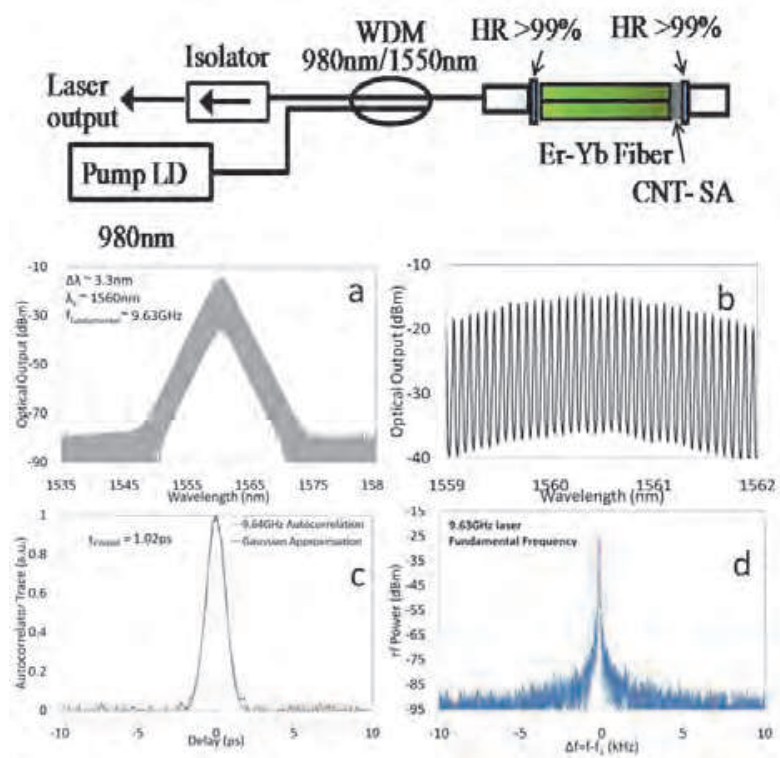

Fig. 8. (a) Set-up for the multi-gigahertz repetition rate laser. (b) Optical output, (c) close up to the longitudinal mode spacing $(\mathrm{d})$ autocorrelator trace (e) rf spectrum.

\section{Ultrafast optical switching using CNT's third order susceptibility}

Considering the vast amount of theoretical and experimental studies on the high third order nonlinearity of CNT now available in literature and the extraordinary evolution of the CNTbased fiber laser technology, the number of reports demonstrating functional CNT devices that exploit the third order susceptibility of CNT is subdued. In this section, we describe some of the most significant advances in the implementation of CNT-based devices 
demonstrating optical switching by wavelength rotation and by four-wave mixing (FWM). This work was pioneered by Y.W. Song et al. and K. Kashiwagi et al., who used CNT-coated D-shape fiber (Song et al., 2007) and a CNT-coated planar lightwave circuit (Kashiwagi et al., 2007) respectively in order to increase the nonlinearity of the devices.

\subsection{Wavelength conversion by polarization rotation}

Tunable wavelength conversion by polarization rotation has been demonstrated using two types of fiber devices, namely a D-shape fiber and a standard SMF fiber with a micro-slot fiber devices inscribed through the core. In this case, the nonlinear process responsible for the polarization rotation is cross-phase modulation (XPM). The concept of optical switching by polarization rotation is described in Figure 9.
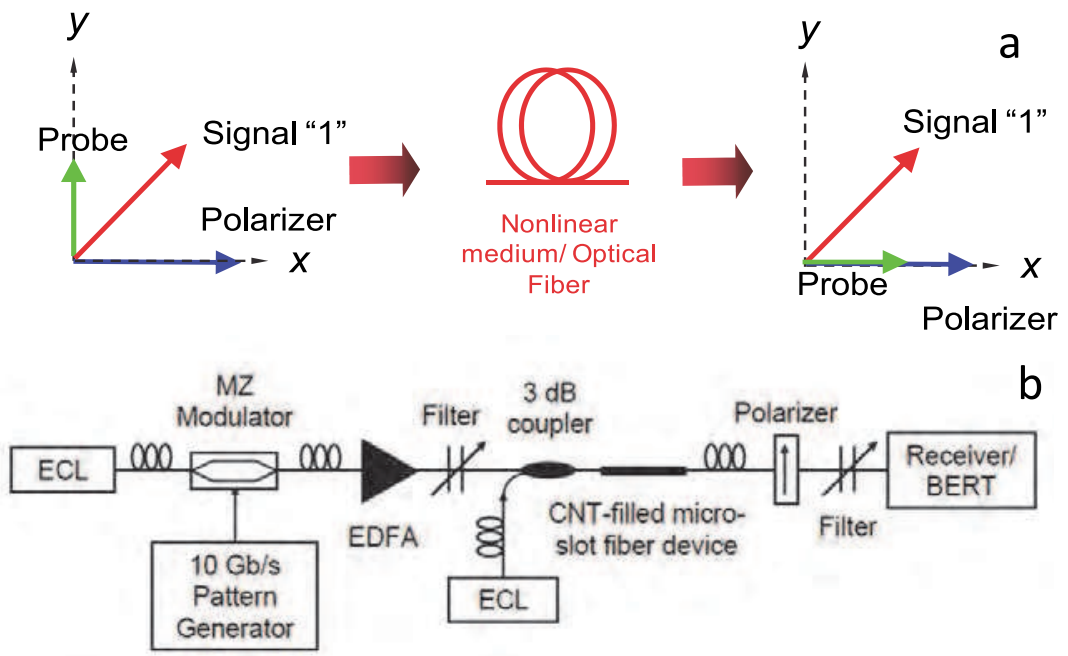

Fig. 9. (a) Depiction of the process of optical switching by polarization rotation. (b) Experimental set-up used for polarization rotation by a micro-slot fiber device (Chow et al., 2009).

Here, we will describe a characteristic polarization rotation experiment by XPM using a CNT filled micro-slot fiber device. Conceptually, the process is the same for similar devices using Dshape or taper fibers. The set up used for this experiment is shown in figure 9 , the pump light polarization is set so in absence of probe light its state of polarization is $45^{\circ}$ with respect to the polarizer, and the probe is set so its state of polarization is $90^{\circ}$ with respect to the polarizer (hence in the absence of pump light the output from the source would be fully suppressed). When both pump and probe co-exist in the nonlinear media (in this case the CNT) the state of polarization of the probe light rotates hence the probe light is received in the detector. In principle, by adjusting the pump light power, it should be possible to transmit through the polarizer $100 \%$ of the probe light. In this particular case, The input signal was a $2^{31-1}$ bits pseudorandom NRZ signal at $10 \mathrm{~Gb} / \mathrm{s}$ amplified by an erbium-doped fiber amplifier (EDFA), an optical bandpass filter was used to eliminate the ASE from the EDFA. The pump and probe were combined through a coupler and launched into the nonlinear fiber device component, which in this case is a $50 \mu \mathrm{m}$-long CNT-filled micro-slot fiber device. For this set-up, the launched signal power and cw light are estimated to be $17 \mathrm{dBm}$ and $-3 \mathrm{dBm}$, respectively. 
The spectrum obtained at the output of the polarizer is shown in Figure 10. In our experiment the input and converted signals are set at $1555.0 \mathrm{~nm}$ and $1545.0 \mathrm{~nm}$, respectively. Fig. 10(b) and 10(c) depicts the close-up spectra with a resolution of $0.01 \mathrm{~nm}$ of the input signal and converted signal, respectively. Note that the converted wavelength is modulated with obvious spectral broadening, showing that wavelength conversion is successfully obtained. In a previous experiment with $\mathrm{cw}$ light only without signal modulation, an on off extinction ratio of $13 \mathrm{~dB}$ of the converted wavelength is obtained. Also, it is worth noting that the converted signal is tunable with similar switching properties. In order to investigate the system performance of the wavelength converter, $10 \mathrm{~Gb} / \mathrm{s}$ bit-error-rate (BER) measurements were performed. Fig. 10(d) plots the output BER against the received optical power. The wavelengths used for input and converted signal are the same with those shown in Fig. 10(a). Fig. 10(e) shows the $10 \mathrm{~Gb} / \mathrm{s}$ eye diagrams of the input signal and the converted signal. The figure shows the results of a 10-nm down-conversion and the power penalty is measured to be $3 \mathrm{~dB}$ at 10-9 BER level. It is believed that one possible source of the power penalty is originated from the insufficient extinction ratio of the converted signal. If the length of the micro-slot and the CNT concentration inside are further increased, higher extinction ratio and lower power penalty are expected.
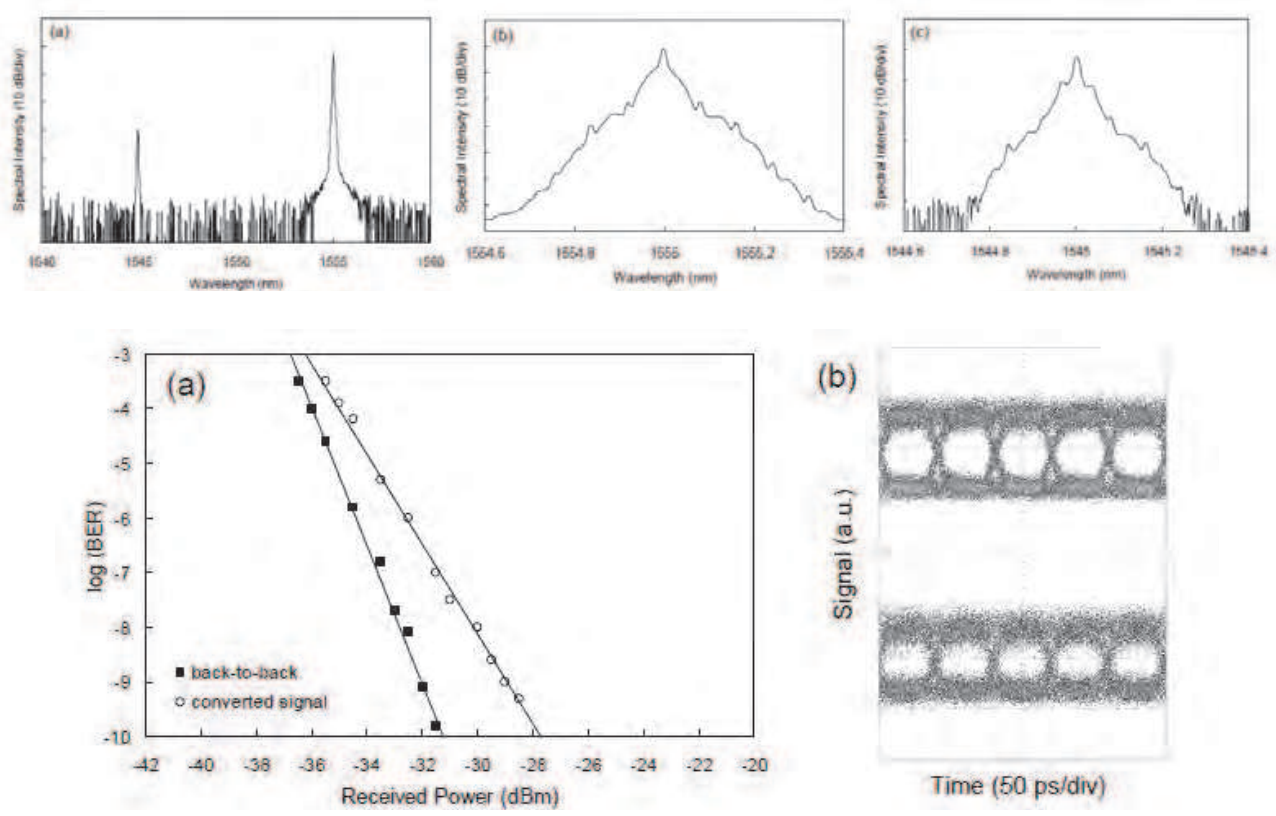

Fig. 10. Experimental results (a) pump and probe signals. Close up of the modulated (b) pump and (c) probe. (d) Bit-error-rate as a function of the received power and (e) eye diagram of the input and received signal.

Similar experiments have been carried out using a $5 \mathrm{~cm}$-long D-shape fiber coated with CNT. In this case the interaction length was three orders of magnitude longer than for the microslots here described, furthermore, the launched pump power was $21 \mathrm{dBm}$ (higher than the $17 \mathrm{dBm}$ used for the micro-slots). However, the power penalty was approximately the same 
$(2.5 \mathrm{~dB})$. These results can be understood considering that the D-shape fiber method in actual fact makes use of evanescent field interaction and therefore the light intensity interacting with the CNT is significantly lower than in the case of the micro-slot fiber devices. Furthermore, it is difficult to fabricate a D-shape with a smooth surface and accurate control of the distance between the fiber and the core. Roughness in the surface leads to increases in the scattering losses.

\subsection{Four-wave-mixing by wavelength conversion}

Four-wave mixing as well as the nonlinear polarization rotation is caused by a propagating media with a high third-order optical nonlinear susceptibility, $\chi^{(3)}$. In this case, when two frequency components propagate through the CNT-device, and additional frequency component is created. An graphic illustration of the process employed is depicted in figure 11(a), two photons of frequency $\omega_{1}$ (pump) interact with a single photon of frequency $\omega_{2}$ (signal) to create an additional component with a frequency of $2 \omega_{1}-\omega_{2}$. FWM is a phasesensitive process and the effect can accumulate over distance if the phase matching conditions are met. In a fiber configuration, phase matching conditions are satisfied if the components' frequencies are close to each other, or nonlinear propagating media exhibits the required chromatic dispersion. FWM is caused by self-phase and cross phase modulation.

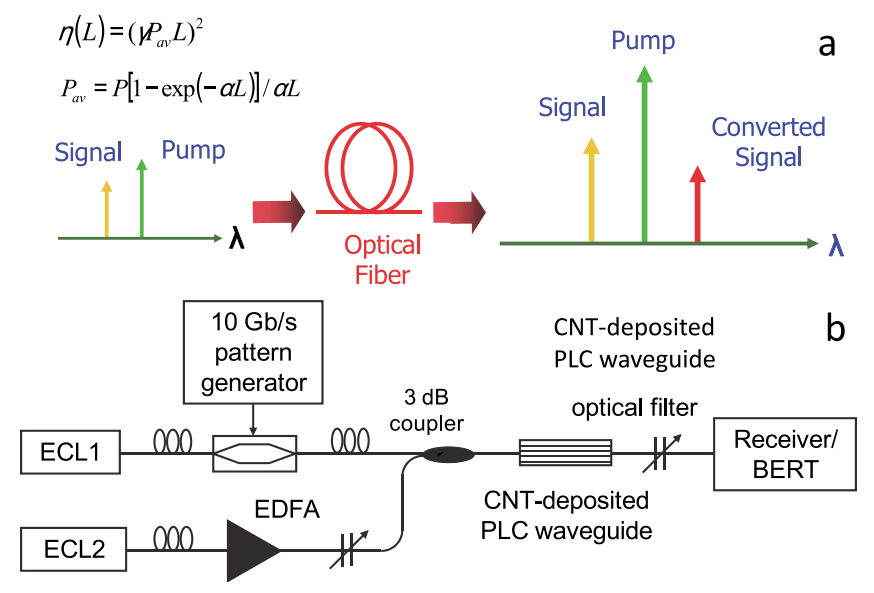

Fig. 11. (a) Depiction of the process of optical switching by four wave mixing FWM). (b) Experimental set-up used for the FWM experiments using a planar lightwave circuit (PLC) (Chow et al., 2010).

FWM employing CNT has been achieved by using a CNT-coated taper fiber and a planar lightwave circuit (PLC). The set-up described in figure 11 corresponds to experiments employing the PLC device. The probe light is combined with a cw pump light from ECL2 through a $3 \mathrm{~dB}$ coupler. The combined light is then launched on a high power EDFA followed by the prepared CNT-deposited tapered fiber. The launched pump power into the fiber is estimated to be around $+27 \mathrm{dBm}$. The amplified light then undergoes FWM effect and idlers with new wavelengths are generated. Figure 12 shows the FWM spectrum obtained after the CNT-deposited tapered fiber. In this experiment, the pump is fixed at $1550.0 \mathrm{~nm}$ and different 
idler wavelengths are obtained by tuning the probe wavelength. FWM conversion efficiency can be defined as the ratio of the idler power to the probe power inside the CNT-deposited tapered fiber. The FWM conversion efficiency can be approximately as shown in equation 4;

$$
\eta(L)=\left(\gamma P_{a v} L\right)^{2}
$$

where $\mathrm{L}$ is the propagation distance through the CNT-deposited region, $\gamma$ is the effective nonlinear coefficient, and $\mathrm{P}$ is the pump power. The effective nonlinear coefficient of the CNT-deposited PLC in our experiment is calculated to be $5.64 \times 10^{5} \mathrm{~W}^{-1} \mathrm{~km}^{-1}$.

The nonlinear properties of the CNT-deposited PLC are used for FWM-based wavelength conversion experiment by modulating the probe light to be a $2^{31}-1$ bits pseudorandom NRZ signal at $10 \mathrm{~Gb} / \mathrm{s}$. The signal is then amplified by a low noise EDFA with amplified spontaneous emissions ASE filtering in order to compensate the insertion loss of the modulator. The signal and the cw pump are then combined and launched into the CNTcoated PLC. As shown in Figure 12, FWM is achieved and the relationship between the conversion efficiency and the input signal wavelength detuning against the fixed pump is shown in Fig. 12 (d). A 3 dB tunning range of $8 \mathrm{~nm}$ is obtained. However, since the length of the device is very short, it is in principle relatively easy to maintain the FWM phase matching condition. Fig. 12(e) shows the bit error rate (BER) as a function of the received optical power with the inset showing the $10 \mathrm{~Gb} / \mathrm{s}$ eye diagrams of the input signal and the converted signal. The figure shows the results of a $3 \mathrm{~nm}$ down-conversion of the input signal with a power penalty of $3 \mathrm{~dB}$ at $10^{-9} \mathrm{BER}$ level.
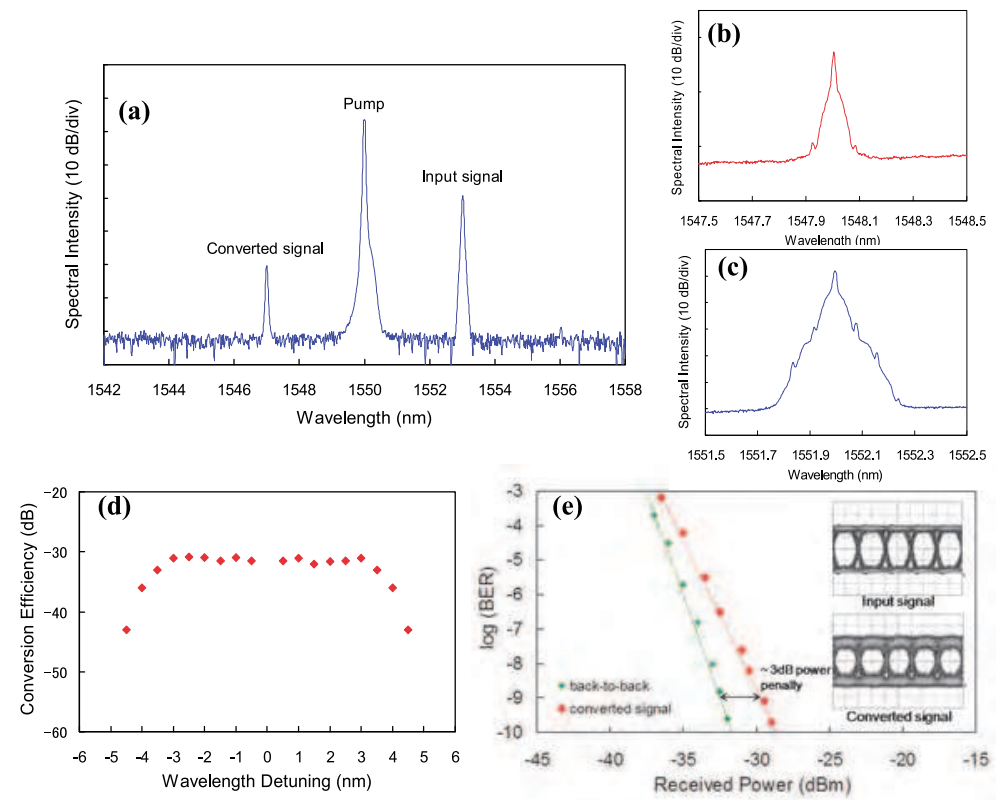

Fig. 12. (a) Optical output showing the pump and converted and input signal, close up of the (b) converted and (c) input signal. (d) Detuning range and (e) Bit error rate as a function of the received power. Inset shows the input and received eye diagrams. 


\section{Current trends and outlook}

Carbon nanotube photonic technology is already a reality with various commercial photonics solution employing CNT-based devices. it has been remarkable the transformation of CNT-based fiber lasers from a scientific curiosity into a fundamental building block in laying the foundations of the next generation fiber laser devices. Recently, graphene has also been considered for saturable absorption applications. Graphene in itself is also a very interesting material with extraordinary potential in several photonic applications, and it is gaining ground over CNT and SESAM devices in part thanks to the knowledge acquired over the years studying CNTs to implement efficient devices. It is still early to say whether these three technologies will over time coexist and be applied depending on the application or one of the three will overtake the others. In the last few years, CNT have been making the transition from a material with very interesting optical properties and potential from a scientific point of view to a position where CNT-SA are an important device in the development of real technological applications and their advantages over competing techniques are utilized. In addition to the results reported in this paper, a number of reports apply CNT to the development of optical sampling systems (Set et al, 2008), frequency combs (Lim et al., 2009), octave spanning supercontinuum generation Kieu et al., 2010), few-cycle pulses fiber lasers (Kieu et al., 2010) and tunable, ultra-broadband fiber laser operation (Wang et al. 2008). CNT-based optical switching on the other hand is still in its infancy, current demonstrations only show modest results and while the very high third order susceptibility combined with the fast optical response is promising, significant developments will need to be made before optical switching by CNT can become a reality in a realistic commercial application.

\section{Conclusion}

In this chapter we described the optical properties of $\mathrm{CNT}$ and their most relevant applications, including saturable absorption for the passive mode-locking of fiber lasers, and optical switching for wavelength conversion have been reviewed.

\section{References}

H. Kataura, Y. Kumazawa, Y. Maniwa, I. Umezu, S. Suzuki, Y. Ohtsuka, and Y. Achiba "Optical properties of single-wall carbon nanotubes," Synth. Met. 103, 2555-2558 (1999)

Margulis, AV, Sizikova, TA: Theoretical study of third order nonlinear optical response of semiconductor carbon nanotube. Physica B, 245, 173-189 (1998)

S. Ijima "Helical microtubules of graphitic carbon", Nature, 354 56-58 (1991)

M. S. Dresselhaus, G. Dresselhaus, and P. Avouris, Eds., Carbon Nanotubes: Synthesis, Structure, Properties, and Application. New York: Springer-Verlag, Berlin, 2001)

P. Avouris, M. Freitag and V. Perebeinos, "Carbon-nanotube Photonics and Optoelectronics", Nature Photonics 2, 341-350 (2008)

S. Y. Set, H. Yaguchi, Y. Tanaka and M. Jablonski, "Ultrafast Fiber Pulsed Lasers Incorporating Carbon Nanotubes," IEEE J. Sel. Top. Quantum Electron. 10, 137 (2004) 
Y.-C. Chen, N.R. Raravikar, L.S. Schadler, P.M. Ajayan, Y.-P. Zhao, T.-M. Lu, G.-C. Wang and X.-C. Zhang, "Ultrafast optical switching properties of single-wall carbon nanotube polymer composites at 1.55 mu m," Appl. Phys. Lett. 81, 975-977 (2002)

X. Liu et al. "Third-order optical nonlinearity of the carbon nanotubes", Appl. Phys. Lett. 74, 164-166, (1999)

R. Saito, M. Fujita, G. Dresselhaus, and M. S Dresselhaus "Electronic structure of chiral graphene tubules" Appl. Phys. Lett. 60, 2204 (1992)

S. Yamashita et al. "Saturable absorbers incorporating carbon nanotubes directly synthesized onto substrates and fibers and their application to mode-locked fiber lasers," Opt. Lett. 29, 1581-1583 (2004)

S. Reich et al., Carbon Nanotubes, Wiley-VCH,2004

Samuli Kivistö, Tommi Hakulinen, Antti Kaskela, Brad Aitchison, David P. Brown, Albert G. Nasibulin, Esko I. Kauppinen, Antti Härkönen, and Oleg G. Okhotnikov, "Carbon nanotube films for ultrafast broadband technology," Opt. Express 17, 23582363 (2009)

F. Wang, A.G. Rozhin, V. Scardaci, Z. Sun, F. Hennrich, I.H. White, W.I. Milne, A.C. Ferrari, "Wideband-tuneable, nanotube mode-locked, fibre laser," Nature Nanotechnology, 3, 738-742 (2008)

Agrawal G.P. “Nolinear Fiber Optics”, Academic Press, 2nd edition 1995

Y. W. Song, S. Yamashita, E. Einarsson, S. Maruyama, "All-fiber pulsed lasers passively mode-locked by transferable vertically aligned Carbon nanotube film," Optics Letters, 32, pp.1399-1401, (2007).

K. Kashiwagi, S. Yamashita, and S. Y. Set, "Optical reflectometry for in-situ monitoring of carbon nanotubes deposition by optical tweezers," Conference on Lasers and Electro Optics (CLEO 2007), no.JThD82, May 2007.

J. W. Nicholson, R. S. Windeler, and D. J. DiGiovanni, "Optically driven deposition of single-walled carbon-nanotube saturable absorbers on optical fiber end-faces," Opt. Express 15, 9176-9183 (2007).

A.G. Rozhin, Y. Sakakibara, S. Namiki, M. Tokumoto, H. Kataura, "Sub-200-fs pulsed erbium-doped fiber laser using a carbon nanotube-polyvinylalcohol mode-locker," Appl. Phys. Lett. 88, 051118 (2006)

T.R. Schibli, K. Minoshima, H. Kataura, E. Itoga, N. Minami, S. Kazaoui, K. Miyashita, M. Tokumoto and Y. Sakakibara, "Ultrashort pulse-generation by saturable absorber mirrors based on polymer-embedded carbon nanotubes," Opt. Express 13, 80258031 (2005)

A.E. Tausenev, E.D. Obraztsova, A.S. Lobach, A.I. Chernov, V.I. Konov, A.V. Konyashchenko, P.G. Kryukov and E.M. Dianov, "Self-mode-locking in erbiumdoped fibre lasers with saturable polymer film absorbers containing single-wall Carbon nanotubes synthesized by the arc discharge method," Quantum Electron. 37 205-208 (2007).

M. Nakazawa, S. Nakahara, T. Hirooka, M. Yoshida, T. Kaino and K. Komatsu, "Polymer saturable absorber materials in the $1.5 \mu \mathrm{m}$ band using polymethyl-methacrylate and polysterene with single-wall carbon nanotubes and their application to a femtosecond laser," Opt. Lett. 31, 915-917 (2006).

S. Uchida, A. Martinez, Y.-W. Song, T. Ishigure, and S. Yamashita, "Carbon nanotube-doped polymer optical fiber," Opt. Lett. 34, 3077-3079 (2009) 
Hasan, T., Sun, Z., Wang, F., Bonaccorso, F., Tan, P. H., Rozhin, A. G. and Ferrari, A. C., "Nanotube-Polymer Composites for Ultrafast Photonics", Advanced Materials, 21, 3874-3899 (2009)

S. Y. Choi, F. Rotermund, H. Jung, K. Oh, and D-I Yeom, "Femtosecond mode-locked fiber laser employing a hollow optical fiber filled with carbon nanotube dispersion as saturable absorber," Opt. Express 17, 21788-21793 (2009)

K. Kieu, M. Mansuripur "Femtosecond laser pulse generation with a fiber taper embedded in carbon nanotube/polymer composite," Opt. Lett. 32, 2242-2244 (2007)

A. Martinez, K. Zhou, I. Bennion and S. Yamashita "In-fiber microchannel device filled with a carbon nanotube dispersion for passive mode-lock lasing" Optics Express, 16, 15425-15430 (2008)

M. E. Fermann and I. Hartl, "Ultrafast fiber laser technology”, IEEE J. Sel. Top. Quantum Electron. $15,191-206$ (2009).

K. Tamura, E. P. Ippen, H. A. Haus, and L. E. Nelson, "77-fs pulse generation from a stretched-pulse mode-locked all-fiber ring laser," Opt. Lett. 18, 1080-1082 (1993)

Z. Sun, T. Hasan, F. Wang, A. G. Rozhin, I. H. White, and A. C. Ferrari, "Ultrafast StretchedPulse Fiber Laser Mode-Locked by Carbon Nanotubes" Nano Res, 3, 404 (2010)

A. Chong, J. Buckley, W. Renninger, and F. Wise, "All-normal-dispersion femtosecond fiber laser," Opt. Express 14, 10095-10100 (2006)

K. Kieu and F. W. Wise, "All-fiber normal-dispersion femtosecond laser," Opt. Express 16, 11453-11458 (2008)

E. J. R. Kelleher, J. Travers, Z. Sun, A. Rozhin, A. Ferrari, S. Popov, and J. Taylor, "Nanosecond-pulse fiber lasers mode-locked with nanotubes," Appl. Phys. Lett. 95, 111108 (2009).

Andreas Schmidt, Simon Rivier, Günter Steinmeyer, Jong Hyuk Yim, Won Bae Cho, Soonil Lee, Fabian Rotermund, Maria C. Pujol, Xavier Mateos, Magdalena Aguiló, Francesc Díaz, Valentin Petrov, and Uwe Griebner, "Passive mode locking of $\mathrm{Yb}: \mathrm{KLuW}$ using a single-walled carbon nanotube saturable absorber," Opt. Lett. 33, 729-731 (2008)

Amos Martinez and Shinji Yamashita, "Multi-gigahertz repetition rate passively modelocked fiber lasers using carbon nanotubes," Opt. Express 19, 6155-6163 (2011)

Y. W. Song, S. Yamashita, C. S. Goh, S. Y. Set, "Passively mode-locked lasers with 17.2-GHz fundamental-mode repetition rate pulsed by Carbon nanotubes," Optics Letters, $.32,430-432$, (2007)

K. K. Chow, S. Yamashita, and Y. W. Song, "A widely tunable wavelength converter based on nonlinear polarization rotation in a carbon-nanotube-deposited D-shaped fiber," Opt. Express 17, 7664-7669 (2009)

K. K. Chow, S. Yamashita, and S. Y. Set, "Four-wave-mixing-based wavelength conversion using a single-walled carbon-nanotube-deposited planar lightwave circuit waveguide," Opt. Lett. 35, 2070-2072 (2010) 


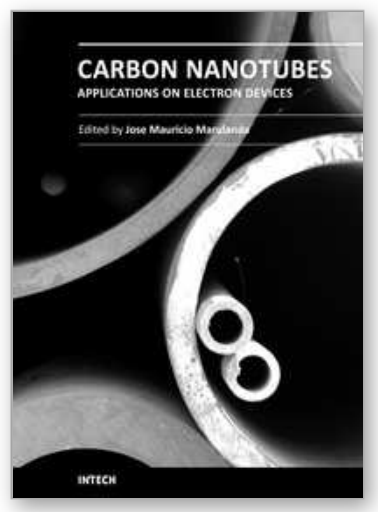

\section{Carbon Nanotubes Applications on Electron Devices}

Edited by Prof. Jose Mauricio Marulanda

ISBN 978-953-307-496-2

Hard cover, 556 pages

Publisher InTech

Published online 01, August, 2011

Published in print edition August, 2011

Carbon nanotubes (CNTs), discovered in 1991, have been a subject of intensive research for a wide range of applications. In the past decades, although carbon nanotubes have undergone massive research, considering the success of silicon, it has, nonetheless, been difficult to appreciate the potential influence of carbon nanotubes in current technology. The main objective of this book is therefore to give a wide variety of possible applications of carbon nanotubes in many industries related to electron device technology. This should allow the user to better appreciate the potential of these innovating nanometer sized materials. Readers of this book should have a good background on electron devices and semiconductor device physics as this book presents excellent results on possible device applications of carbon nanotubes. This book begins with an analysis on fabrication techniques, followed by a study on current models, and it presents a significant amount of work on different devices and applications available to current technology.

\section{How to reference}

In order to correctly reference this scholarly work, feel free to copy and paste the following:

Amos Martinez and Shinji Yamashita (2011). Carbon Nanotube-Based Photonic Devices: Applications in Nonlinear Optics, Carbon Nanotubes Applications on Electron Devices, Prof. Jose Mauricio Marulanda (Ed.), ISBN: 978-953-307-496-2, InTech, Available from: http://www.intechopen.com/books/carbon-nanotubesapplications-on-electron-devices/carbon-nanotube-based-photonic-devices-applications-in-nonlinear-optics

\section{INTECH}

open science | open minds

\section{InTech Europe}

University Campus STeP Ri

Slavka Krautzeka 83/A

51000 Rijeka, Croatia

Phone: +385 (51) 770447

Fax: +385 (51) 686166

www.intechopen.com

\section{InTech China}

Unit 405, Office Block, Hotel Equatorial Shanghai

No.65, Yan An Road (West), Shanghai, 200040, China

中国上海市延安西路65号上海国际贵都大饭店办公楼 405 单元

Phone: +86-21-62489820

Fax: $+86-21-62489821$ 
(C) 2011 The Author(s). Licensee IntechOpen. This chapter is distributed under the terms of the Creative Commons Attribution-NonCommercialShareAlike-3.0 License, which permits use, distribution and reproduction for non-commercial purposes, provided the original is properly cited and derivative works building on this content are distributed under the same license. 